\title{
A SUMMATION MATRIX WITH A GOVERNOR
}

\author{
GEORGE PIRANIAN
}

1. Introduction and summary. The present paper describes a new method of summation with a triangular matrix. The innovation consists in the fact that the elements in the $n$th row of the matrix are constructed from the first $n$ terms of the series to be summed. The dependence of the matrix on the series is such as to allow fairly rapid convergence of the summation process in the case of "well-behaved" series, yet to make the method applicable to some series whose partial sums oscillate violently. The method is included in the Abel method.

2. Summability $(G)$. Corresponding to any formal series $\sum_{n=0}^{\infty} a_{n}$ we form the sequence $\left\{S_{n}\right\}$ determined by the relation

$$
S_{n}=\frac{\sum_{r=0}^{n} s_{r} \sum_{k=0}^{n-r}\left|a_{k}\right|}{\sum_{r=0}^{n} \sum_{k=0}^{r}\left|a_{k}\right|},
$$

where $s_{r}=\sum_{k=0}^{r} a_{k}(r=0,1,2, \cdots)$ (the right member of equation (1) has meaning except when $a_{k}=0(k=0,1,2, \cdots, n)$, in which case we write $S_{n}=0$ ).

DEFINITION. If

$$
\lim _{n \rightarrow \infty} \sum_{k=0}^{n}\left|a_{k}\right| / \sum_{r=0}^{n} \sum_{k=0}^{r}\left|a_{k}\right|=0
$$

and

$$
\lim _{n \rightarrow \infty} S_{n}=S,
$$

the series $\sum a_{n}$ is summable $(G)$ to $S$; if we wish to be more explicit, we say that the series is summable $\left(G, \sum_{k=0}^{n-r}\left|a_{k}\right|\right)$ to $S$ (read: summable with the governor $\left.\sum_{k=0}^{n-r}\left|a_{k}\right|\right)$.

It is evident that equation (1) can be written in the form

$$
S_{n}=\sum_{r=0}^{n} s_{r} p_{n-r} / P_{n}
$$

where $p_{k}=\sum_{h=0}^{k}\left|a_{h}\right|$ and $P_{n}=\sum_{k=0}^{n} p_{k}$. In other words, equation (1) represents a Nörlund transformation $[3]^{1}$ with positive increasing co-

Presented to the Society, April 27, 1946; received by the editors March 13, 1946.

1 Numbers in brackets refer to the references cited at the end of the paper. 
efficients, provided that condition (A) is satisfied.

The notation of the last paragraph will be used throughout this paper. Where more than one series is under discussion, symbols such as $S_{n}\{a\}, P_{n}\{a\}$ will be used to indicate quantities associated with the series $\sum a_{n}$.

THEOREM 1. Summability $(G)$ is consistent with every Nörlund method with positive coefficients.

Corollary. Summability $(G)$ is consistent with the Cesdro methods of all orders.

Theorem 2. Summability $(G)$ is included in the Abel method.

THEOREM 3. If the series $\sum a_{n}, \sum b_{n}, \sum\left(a_{n}+b_{n}\right)$ are summable $(G)$ to $A, B$, and $C$, respectively, then $A+B=C$.

Theorem 1 follows from the fact that all Nörlund methods with positive coefficients are consistent with respect to each other [2], and its corollary from the fact that all Cesàro methods of integral order are Nörlund methods [1]. Theorems 2 and 3 are consequences of Silverman and Tamarkin's theorem [4] to the effect that every Nörlund method with positive coefficients is contained in the Abel method.

LemMA. A necessary condition for condition A to be satisfied is that $\lim \inf _{n \rightarrow \infty}\left|a_{n}\right| / p_{n}=0 ; a$ sufficient condition is that $\lim _{n \rightarrow \infty} a_{n} / p_{n}=0$.

To prove our lemma, we write,

$$
p_{n} / P_{n}=p_{t} / P_{n}+\left(p_{n}-p_{t}\right) / P_{n}=p_{t} / P_{n}+\frac{p_{n}-p_{t}}{P_{n}-P_{t}}\left(1-P_{t} / P_{n}\right) \text {, }
$$

and we recall that if $b_{i}, c_{i}$ are non-negative numbers such that $H \leqq b_{i} / c_{i} \leqq K \quad(i=1,2, \cdots, s)$, then $H \leqq \sum_{i=1}^{s} b_{i} / \sum_{i=1}^{s} c_{i} \leqq K$. If $\lim \inf _{n \rightarrow \infty}\left|a_{n}\right| / p_{n}>h>0$, the value of the fraction $\left(p-p_{t}\right) /\left(P_{n}-P_{t}\right)$ exceeds $h$ if $t$ is sufficiently large and $n$ is greater than $t$. Since $p_{r}$ is a non-decreasing function of $r$, the values of $p_{t} / P_{n}$ and $P_{t} / P_{n}$ tend to zero as $n$ becomes large, and condition (A) is not satisfied.

If on the other hand $\lim _{n \rightarrow \infty} a_{n} / p_{n}=0$, a similar argument shows that condition (A) is satisfied, and the proof of the lemma is complete.

THEOREM 4. The method of summability $(G)$ includes the method of convergence.

This theorem follows from the preceding lemma and the fact that every Nörlund method includes the method of convergence (a trivial exception to the theorem exists: if $a_{n}=0(n=0,1,2, \cdots), \lim _{n \rightarrow \infty} S_{n}$ 
$=0$, but condition (A) fails to be satisfied, and a slight modification of our definition is necessary for the series $\sum a_{n}$ to be summable $(G)$ ).

It should here be remarked that the condition $\lim _{n \rightarrow \infty} a_{n} / p_{n}=0$ is not necessary for condition (A) to be satisfied, as can be seen from the following example: Let

$$
\begin{array}{rlrl}
a_{0} & =0, \\
\left|a_{n}\right| & =1 & (n \neq 0,1,2,4, \cdots), \\
\left|a_{n}\right| & =p_{n-1} & (n=1,2,4, \cdots) .
\end{array}
$$

We demonstrate, by induction, that

$$
p_{n} \leqq n \log _{2} n \quad(n=1,2,3, \cdots),
$$

where the subscript 2 indicates the base of the logarithm. Suppose that

$$
p_{r} \leqq r \log _{2} r
$$

where $r=2^{h}$ ( $h$ a certain positive integer). Then

$$
\begin{aligned}
p_{r+t} & \leqq r \log _{2} r+t<(r+t) \log _{2}(r+t) \quad(t<r), \\
p_{2 r-1} & \leqq r \log _{2} r+r-1, \\
p_{2 r} & <2 r \log _{2} r+2 r=2 r \log _{2}(2 r) ;
\end{aligned}
$$

that is, the inequality holds when $r=2^{h}+1,2^{h}+2, \cdots, 2^{h+1}$. Since the inequality holds when $r=1$, and since

$$
P_{n} \geqq \sum_{r=3}^{n}(r-2)=(n-2)(n-1) / 2,
$$

it follows that

$$
p_{n} / P_{n} \leqq \frac{2 n \log _{2} n}{(n-2)(n-1)},
$$

that is, condition (A) is satisfied while $\lim \sup _{n \rightarrow \infty}\left|a_{n}\right| / p_{n}=1 / 2$.

3. Special properties. In this section we consider some of the properties of the sequence $S_{n}$ that are of interest even when the sequence fails to converge.

THEOREM 5. If $b_{n}=h a_{n}(n=0,1,2, \cdots)$ where $h$ is a constant, then $S_{n}\{b\}=h S_{n}\{a\}$.

This theorem follows immediately from the fact that $p_{n}\{b\}$ $=|h| p_{n}\{a\}, P_{n}\{b\}=|h| P_{n}\{a\}$, and $s_{n}\{b\}=h s_{n}\{a\}$.

The next theorem concerns the effect of altering the values of a finite number of terms of the series $\sum a_{n}$. Since summability $(G)$ is a 
regular method, it suffices to consider the case of nonconvergent series.

If $b_{n}=a_{n}(n \neq i)$ and $b_{i}=a_{i}+h$,

$$
\begin{aligned}
\sum_{r=0}^{n} s_{r}\{b\} p_{n-r}\{b\}= & \sum_{r=0}^{n} s_{r}\{a\} p_{n-r}\{b\}+h \sum_{r=i}^{n} p_{n-r}\{b\} \\
= & \sum_{r=0}^{n} s_{r}\{a\} p_{n-r}\{a\} \\
& +\left(\left|a_{i}+h\right|-\left|a_{i}\right|\right) \sum_{r=0}^{n-i} s_{r}\{a\}+h P_{n-i}\{b\}
\end{aligned}
$$

it follows that

$$
\begin{aligned}
S_{n}\{b\}= & S_{n}\{a\} P_{n}\{a\} / P_{n}\{b\}+\left(\left|a_{i}+h\right|-\left|a_{i}\right|\right) \sum_{r=0}^{n-i} s_{r}\{a\} / P_{n}\{b\} \\
& +h\left(1-\sum_{n-i+1}^{n} p_{r}\{b\} / P_{n}\{b\}\right) .
\end{aligned}
$$

If condition (A) is satisfied by the series $\sum a_{n}$,

$$
\begin{aligned}
P_{n}\{b\} & =P_{n}\{a\}+(n-i+1)\left(\left|a_{i}+h\right|-\left|a_{i}\right|\right) \\
& =\left(1+\epsilon_{n}\right) P_{n}\{a\},
\end{aligned}
$$

where $\lim _{n \rightarrow \infty} \epsilon_{n}=0$ (nonconvergence of the series $\sum a_{n}$ implies that $\lim _{n \rightarrow \infty} p_{n}\{a\}=\infty$ and therefore that $\left.\lim _{n \rightarrow \infty} P_{n}\{a\} / n=\infty\right)$. From this it follows that the series $\sum b_{n}$ also satisfies condition (A), and that

$$
S_{n}\{b\}=\left(1+\epsilon_{n}^{\prime}\right) S_{n}\{a\}+\left(1+\epsilon_{n}^{\prime \prime}\right) h+\left(\left|a_{i}+h\right|-\left|a_{i}\right|\right) \theta_{n}
$$

where $\lim _{n \rightarrow \infty} \epsilon_{n}^{\prime}=0=\lim _{n \rightarrow \infty} \epsilon_{n}^{\prime \prime}$ and $\left|\theta_{n}\right| \leqq \max _{r} \leqq n-i\left|s_{r}\{a\}\right| / p_{r+i}\{b\}$. Imposing the further requirement that $\lim _{n \rightarrow \infty} \theta_{n}=0$, we now obtain the following result:

THEOREM 6. If the series $\sum a_{n}$ satisfies condition (A); if

$$
\lim _{n \rightarrow \infty} \sum_{r=0}^{n} s_{r}\{a\} / P_{n}\{a\}=0
$$

and if

then

$$
\begin{aligned}
b_{n} & =a_{n} & \left(n \neq n_{1}, n_{2}, \cdots, n_{k}\right), \\
b_{n_{i}} & =a_{n_{i}}+h_{i} & (i=1,2, \cdots, k) ;
\end{aligned}
$$

$$
S_{n}\{b\}=\left(1+\epsilon_{n}\right) S_{n}\{a\}+\sum_{i=1}^{k} h_{i}+\eta_{n},
$$

where $\lim _{n \rightarrow \infty} \epsilon_{n}=0=\lim _{n \rightarrow \infty} \eta_{n}$. 
COROLLARY 1. If the set of partial sums $s_{n}\{a\}$ is bounded, $\lim \left(S_{n}\{b\}\right.$ $\left.-S_{n}\{a\}\right)=\sum_{i=1}^{k} h_{i}$.

CoROLlaRy 2. If the series $\sum a_{n}$ satisfies conditions (A) and (B) and is (is not) summable $(G)$, every series differing from it in a finite number of terms only is (is not) summable $(G)$.

THEOREM 7. If

$$
\begin{array}{rlrl}
b_{n} & =a_{n} & & (n=0,1, \cdots, i-1), \\
b_{i} & =0, & \\
b_{n}=a_{n-1} & (n=i+1, i+2, \cdots)
\end{array}
$$

and the series $\sum a_{n}$ satisfies condition (A), then

$$
S_{n}\{b\}-S_{n-2}\{a\}=\epsilon_{n} S_{n-2}\{a\}+\epsilon_{n}^{\prime}
$$

where $\lim _{n \rightarrow \infty} \epsilon_{n}=0=\lim _{n \rightarrow \infty} \epsilon_{n}^{\prime}$.

This theorem asserts that the insertion of a finite number of terms whose value is zero into a series satisfying condition (A) cannot destroy or bring into existence summability $(G)$ for the series or change the value of the sum $(G)$ of the series. A stronger theorem is of course desirable; we may ask, for example, whether there exists an infinite sequence of integers $\left\{n_{i}\right\}$ such that the insertion of a term of value zero after each of the terms $a_{n_{i}}$ in any series $\sum a_{n}$ satisfying condition A cannot destroy or bring into existence summability $(G)$ for the series or change the value of the sum $(G)$ of the series.

To prove Theorem 7, we observe that (for $n>2 i$ )

$$
P_{n}\{b\}=P_{n-2}\{a\}+p_{n-1}\{a\}+p_{i-1}\{a\}
$$

and

$$
\begin{aligned}
\sum_{r=0}^{n} s_{r}\{b\} & p_{n-r}\{b\} \\
& =\sum_{r=0}^{i-1} s_{r}\{b\} p_{n-r}\{b\}+s_{i}\{b\} p_{n-i}\{b\}+\sum_{r=i+1}^{n} s_{r}\{b\} p_{n-r}\{b\} \\
& =\sum_{r=0}^{i-1} s_{r}\{b\} p_{n-r}\{b\}+s_{i}\{b\} p_{n-i}\{b\}+\sum_{r=i}^{n-1} s_{r}\{a\} p_{n-r-1}\{b\} \\
& =\sum_{r=0}^{n-2} s_{r}\{a\} p_{n-2-r}\{a\}+\eta_{n} P_{n}\{b\} \quad\left(\lim _{n \rightarrow \infty} \eta_{n}=0\right) .
\end{aligned}
$$

Division of $\sum_{r=0}^{n} s_{r}\{b\} p_{n-r}\{b\}$ by $P_{n}\{b\}$ gives the desired result.

The following theorem regarding the summability $(G)$ of certain 
alternating series provides a first indication of the strength of the method $(G)$ :

THEOREM 8. If $f(z)$ is a polynomial in $z$ with real coefficients, then the series $\sum_{n=0}^{\infty}(-1)^{n} f(n)$ is summable $(G)$.

We assume, first, that $f(z) \geqq 0$ when $z=0,1,2, \ldots$. If $f(z)$ is of degree $q$, the quantities $p_{n}$ and $P_{n}$ are then polynomials in $n$ of degree $q+1$ and $q+2$, respectively. We shall show that for each $n$ the sum $\sum_{r=0}^{n} s_{r} p_{n-r}$ can be expressed as one of two polynomials of degree $q+2$ or less in $n$, and that the leading terms of the two polynomials have identical coefficients.

Writing

$$
\sum_{r=0}^{n} s_{r} p_{n-r}=\sum_{r=0}^{n} \sum_{k=0}^{r} a_{k} p_{n-r}=\sum_{k=0}^{n} a_{k} \sum_{r=k}^{n} p_{n-r}=\sum_{k=0}^{n} a_{k} P_{n-k},
$$

and $P_{n}=f_{2}(n)$, we have the relation

$$
\sum_{r=0}^{n} s_{r} p_{n-r}=\sum_{r=0}^{n}(-1)^{r} f(r) f_{2}(n-r) .
$$

Now, if $g(z)$ is holomorphic in the region $-1 / 2 \leqq x \leqq n+1 / 2(z=x+i y)$ and if, for some pair of constants $(C, \alpha)$ with $\alpha<1$, the inequality $|g(z)|<C e^{\alpha|y|}$ holds in this region, then [2, p. 53]

$$
\sum_{r=0}^{n}(-1)^{r} g(r)=\frac{1}{2 i} \int_{n+1 / 2-i \infty}^{n+1 / 2+i \infty} \frac{g(z)}{\sin \pi z} d z-\frac{1}{2 i} \int_{-1 / 2-i \infty}^{-1 / 2+i \infty} \frac{g(z)}{\sin \pi z} d z .
$$

In our case, this identity takes the form

$$
\begin{aligned}
& \sum_{r=0}^{n} s_{r} p_{n-r}=\frac{1}{2 i} \int_{n+1 / 2-i \infty}^{n+1 / 2+i \infty} \frac{f(z) f_{2}(n-z)}{\sin \pi z} d z-\frac{1}{2 i} \int_{-1 / 2-i \infty}^{-1 / 2+i \infty} \frac{f(z) f_{2}(n-z)}{\sin \pi z} d z \\
& =\frac{1}{2} \int_{-\infty}^{\infty} \frac{(-1)^{n} f(n+1 / 2+i t) f_{2}(-1 / 2-i t)+f(-1 / 2+i t) f_{2}(n+1 / 2-i t)}{\cosh \pi t} d t,
\end{aligned}
$$

and the theorem is proved for the case where $f(z) \geqq 0$ when $z=0,1$, $2, \cdots$.

If the polynomial $f(z)$ is negative for some of the values $z=0,1$, $2, \cdots, k-1$, and positive for $z=k, k+1, \cdots$, we consider first the series

$$
\sum_{n=0}^{\infty}(-1)^{n+k} f(n+k)=\sum_{n=k}^{\infty}(-1)^{n} f(n) .
$$

This series is summable $(G)$, and by Theorem 7 it is still summable $(G)$ 
after $k$ terms of value zero are prefixed to it. It follows from Theorem 6 that the series $\sum(-1)^{n} f(n)$ is summable, and the proof of Theorem 8 is complete.

We note, incidentally, that in the special case under consideration in Theorem 8 ,

$$
\lim _{n \rightarrow \infty} S_{n}=\frac{1}{2} \int_{-\infty}^{\infty} \frac{f(-1 / 2+i t)}{\cosh \pi t} d t .
$$

If $f(z)$ is non-negative $(z=0,1,2, \cdots)$, this is obvious from the fact that in the quantity $\sum_{r=0}^{n} s_{r} p_{n-r}$ the term of highest degree in $n$ is the same as in the quantity

$$
\frac{1}{2} \int_{-\infty}^{\infty} \frac{f(-1 / 2+i t) f_{2}(n)}{\cosh \pi t} d t .
$$

In the more general case, we choose an arbitrary polynomial $g(z)$ so that $f(z)+g(z) \geqq 0, g(z) \geqq 0(n=0,1,2, \cdots)$. Since the two series $\sum(-1)^{n}[f(n)+g(n)]$ and $\sum(-1)^{n} g(z)$ are summable to

and

$$
\frac{1}{2} \int_{-\infty}^{\infty} \frac{f(-1 / 2+i t)+g(-1 / 2+i t)}{\cosh \pi t} d t
$$

$$
\frac{1}{2} \int_{-\infty}^{\infty} \frac{g(-1 / 2+i t)}{\cosh \pi t} d t
$$

respectively, the result follows at once from Theorem 3 .

The following proposition is now a consequence of Theorem 2:

THEOREM 9. If $f(z)$ is a polynomial with real coefficients,

$$
\lim _{x \rightarrow-1+0} \sum_{n=0}^{\infty} x^{n} f(n)=\frac{1}{2} \int_{-\infty}^{\infty} \frac{f(-1 / 2+i t)}{\cosh \pi t} d t .
$$

4. An unsolved problem. The method of the present paper can be extended to matrices with governors other than $\sum_{\substack{n=0 \\ n-r}}\left|a_{k}\right|$. Any relation of the form

$$
p_{n}=p\left(a_{0}, a_{1}, \cdots, a_{n}\right)
$$

or, more generally,

$$
p_{n}=p\left(n ; a_{0}, a_{1}, \cdots\right)
$$

determines a Nörlund method, corresponding to each series $\sum a_{n}$ satisfying the appropriate analogue of condition (A). It would obviously be of value to know a simple relation of the form (2) or (3) generating 
a "universal Nörlund method $\left(G^{*}\right)$," that is, a method which sums the series $\sum a_{n}$ whenever the series is summable by any Nörlund method.

\section{REFERENCES}

1. E. Cesàro, Sur la multiplication des sêries. Bull. Sci. Math. (2) vol. 14 (1890) pp. 114-120.

2. E. Lindelöf, Le calcul des résidues et ses applications a la théorie des fonctions, 1905.

3. N. E. Nörlund, Sur une application des fonctions permutables, Lunds Universitets Årsskrift N. F. Afdelning 2 vol. 16 (1920).

4. L. L. Silverman and J. D. Tamarkin, Generalization of Abel's theorem. Math. Zeit. vol. 29 (1928) pp. 161-170.

UNIVERSITY OF MichigAN 